\title{
C3-BIOECONOMY
}

Circular and Sustainable Bioeconomy

\section{La Bioeconomía como línea estratégica del Campus de Excelencia Internacional Agroalimentario, ceiA3}

\author{
M. Dolores de Toro-Jordano ${ }^{1}$
}

Autor de Correspondencia: gerente@ceia3.es

\section{Resumen:}

La concienciación sobre la importancia de la Bioeconomía y el fomento de la transferencia para promover avances hacia una economía circular en los sistemas productivos vinculados con la agroalimentación precisa de la implementación de acciones y estrategias, a nivel territorial y regional, y la implicación de diferentes agentes que sirvan de tractor y dinamicen iniciativas con un enfoque botón-up y multi-actor. Aquí es donde el Campus de Excelencia Internacional Agroalimentario, ceiA3, a través de su estrategia bioeconomía, alineada con la RIS3 andaluza, se posiciona como un instrumento clave para la región de Andalucía. El campus promueve la divulgación científica, la concienciación de la sociedad y del tejido productivo en materia de Bioeconomía, asimismo favorece el alineamiento de las instituciones integrantes del campus con las políticas en ese ámbito, dinamizando la interacción entre Universidades y entre grupos de investigación así como su interconexión con el sector agroalimentario para dar respuesta a las demandas específicas del mismo en bioeconomía tanto el formación, capacitación y docencia como en I+D y transferencia a través de un modelo de innovación abierta cuádruple hélice.

Palabras clave: Bioeconomía, RIS3, Agroalimentario, Transferencia, Divulgación

\section{Bioeconomy as a strategic line of the Agri-food Campus of International Excellence, ceiA3}

\author{
M. Dolores de Toro-Jordano ${ }^{1}$
}

\section{Abstract:}

Awareness of the importance of the Bioeconomy and the promotion of its transfer to promote progress towards a circular economy in productive systems linked to agri-food calls for the implementation of a series of actions and strategies at the territorial and regional levels, and the involvement of different agents serving as driving forces and energising initiatives, with a bottomup and multi-actor approach. This is where the Agri-food Campus of International Excellence, ceiA3, through its Bioeconomy strategy, aligned with the Andalusian RIS3, stands as a key instrument for the Andalusian region. The campus promotes scientific dissemination and awareness of the Bioeconomy in society and the productive fabric while favouring the alignment of the institutions that make up the campus with policies in this field, stimulating interaction between

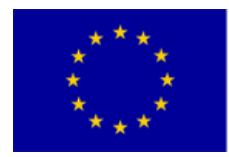

UNION EUROPEA

PROYECTO COFINANCIADO POR EL FONDO EUROPEO DESARR
(FEDER) (FEDER)
Una manera de hacer Europa

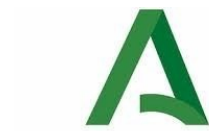

Junta de Andalucía
Andalucía

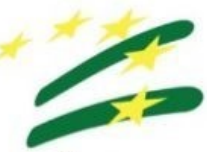

se mueve con Europa 
universities and research groups, as well their interconnection with the agri-food sector, to respond to its specific Bioeconomy-related demands in relation to education, training and teaching, as well as in R\&D and transfer through a quadruple helix open innovation model.

Keywords: Bioeconomy, RIS3, Agri-food, Transfer, Dissemination

'Campus de Excelencia Internacional Agroalimentario (ceiA3), Córdoba (ESPAÑA), correo electrónico: gerente@ceia3.es

\section{MARCO CONCEPTUAL Y POLÍTICO}

La bioeconomía es la producción, utilización y conservación de los recursos biológicos, incluidos los conocimientos relacionados, la ciencia, la tecnología y la innovación, para proporcionarle información, productos, procesos y servicios a todos los sectores económicos, con el objetivo de avanzar hacia una economía sostenible (FAO, 2018).

En Europa tras una primera estrategia en materia de Bioeconomía del año 2012, en noviembre de 2019, el Consejo adoptó sus conclusiones sobre la Estrategia de Bioeconomía actualizada tituladas «Una bioeconomía sostenible para Europa: reforzar la conexión entre la economía, la sociedad y el medio ambientel.

Tal y como se define en la Estrategia Europea de Bioeconomía "La bioeconomía abarca todos los sectores y sistemas basados en los recursos biológicos (animales, plantas, microorganismos y biomasa derivada, incluidos los residuos orgánicos), sus funciones y principios. Incluye, constituyendo un nexo entre ellos, los ecosistemas terrestres y marinos y los servicios que prestan; todos los sectores de producción primaria que utilizan y producen recursos biológicos (agricultura, silvicultura, pesca y acuicultura); y todos los sectores económicos e industriales que utilizan recursos y procesos biológicos para producir alimentos, piensos, bioproductos, energía y servicios. Para poder tener éxito, la bioeconomía europea debe articularse en torno a la sostenibilidad y la circularidad. Ello impulsará la renovación de las industrias, la modernización de 
los sistemas de producción primaria y la protección del medio ambiente, y potenciará asimismo la biodiversidad (Comisión Europea, 2018).

En línea con Europa en el ámbito nacional se dinamiza en 2016 la Estrategia Española de Bioeconomía "Horizonte 2030" donde cabe destacar como objetivos estratégicos:

- Mejorar la competitividad e internacionalización de las empresas existentes.

- Nuevas actividades económicas y nuevos puestos de trabajo con nuevos desarrollos científicos y tecnológicos.

- Posicionamiento de Bioeconomía como área de innovación estratégica basada en el conocimiento.

- Alcanzar potencial de desarrollo en 15 años.

El ámbito de la estrategia "incorpora como sectores objeto de atención al agroalimentario, integrado por la agricultura, ganadería, pesca, acuicultura y elaboración y comercialización de alimentos, como uno de los que está actuando como motor de nuestras exportaciones, al sector forestal y de derivados de la madera, al de los bioproductos industriales, obtenidos con o sin una transformación bioquímica o biológica de la materia orgánica generada por nuestra sociedad, y no utilizada en el consumo humano y animal, así como al de la bioenergía obtenida de la biomasa o el de los servicios asociados a los entornos rurales".

A nivel regional, en la Estrategia de Bioeconomía Circular de Andalucía (Junta de Andalucía, 2018), aprobada en el año 2018, los objetivos estratégicos marcados son:

- Mejorar la sostenibilidad y competitividad de los sectores agroalimentario, pesquero, y forestal, fomentando el uso de prácticas innovadoras que favorezcan y desarrollen una economía circular; 
- Impulsar la competitividad de las industrias que trabajen con recursos biológicos, fomentando la innovación, la generación de conocimiento y la transferencia de tecnología;

- Fomentar la reutilización de los recursos, agua, gases, nutrientes y el aprovechamiento de los residuos y restos vegetales para obtener otros productos, usos o energías;

- Favorecer la investigación, innovación y la cualificación relacionadas con la bioeconomía y

- Reforzar la coordinación interadministrativa, y las sinergias con otros planes y programas de trabajo de distinto ámbito.

Por último, cabría destacar como mensajes clave del Comunicado de la Cumbre Mundial de Bioeconomía 2018 la petición urgente de aumentar el diálogo y colaboración en materia de sostenibilidad en I+D y en capacitación: "con miras a los grandes desafíos de la sociedad, pedimos con urgencia un aumento en el diálogo multilateral y la colaboración en I + D en bioeconomía, en particular investigación sobre sostenibilidad y cambio global, gobernanza y creación de capacidades" (Comunicado Cumbre Global de Bioeconomía, 2018).

\section{2. ceiA3, ROL ESTRATÉGICO HACIA UN TEJIDO AGROALIMENTARIO SOSTENIBLE}

\subsection{Programa Campus de Excelencia Internacional}

Tras esta primera aproximación al marco conceptual de la bioeconomía y a la importancia de promover sistemas productivos sostenibles procede introducir el sentido del programa Campus de Excelencia Internacional, se trata de una iniciativa promovida en 2008 por el Gobierno de España en el ámbito del proceso de modernización de la universidad española que "se dirige hacia la 
agregación estratégica de universidades, de instituciones relacionadas con el Triángulo del Conocimiento, y del sector productivo, que actuando en un entorno o campus especializado, busquen su referencia y posicionamiento en el contexto internacional". Tenía como objetivos el reconocimiento y aval de aquellos proyectos de campus de referencia internacional en docencia, innovación y transferencia, investigación y la interconexión de la universidad con el sector productivo.

\subsection{Campus de excelencia internacional agroalimentario, ceiA3}

En 2009, desde Andalucía, 5 Universidades, la de Córdoba, Almería, Cádiz, Huelva y Jaén promovieron la iniciativa conjunta de Campus de Excelencia Internacional resultado de la agregación de estas 5 universidades coordinados por la Universidad de Córdoba y con especialización en Agroalimentación: el Campus de Excelencia Internacional Agroalimentario, ceiA3, cuyo fin es favorecer la interconexión universidad-empresa, formando profesionales cualificados y acordes con las necesidades del sector, y apoyando su transformación hacia un modelo sostenible.

Desde su puesta en marcha el proyecto se fortaleció con acciones de transferencia, emprendimiento, financiación de becas y ayudas, fomento de las infraestructuras, la colaboración de CSIC e IFAPA como centros asociados, y se impulsó con la creación del consorcio ceiA3 como órgano de gestión del campus.

Por ello y desde el ceiA3 tiene como principal objeto y principio fundacional favorecer el vínculo de la universidad con el sector agroalimentario andaluz para promover su profesionalización en materia de formación y capacitación y su innovación mediante transferencia del conocimiento.

La concepción del ceiA3 como elemento agregador de instituciones y con especialización en agroalimentación motiva que uno de sus principales retos sea su alineamiento tanto con la estrategia de especialización inteligente 
de Andalucía (RIS3) como con las principales estrategias y políticas sectoriales transversales para posicionarse como un elemento clave en el diseño de las mismas.

\subsection{Potencial del ceiA3 en el ámbito de la Bioeconomía}

Las Universidades que componen el ceiA3 imparten docencia reglada agroalimentaria que se adscribe al campus teniendo un amplio porcentaje de ella un peso específico importante en contenidos docentes de áreas relacionadas con la Bioeconomía en 24 de los 100 grados, 18 de los 80 másteres, así como en cursos de especialización como los Training Network Courses del ceiA3 en los que se ha priorizado la bioeconomía en sus 3 últimas convocatorias.

El Campus integrado por 5 universidades y 2 centros asociados conforman un potencial en I+D compuesto por 302 grupos de investigación y cerca de 4.000 investigadores estructurados en 7 clústeres de relevancia para la bioeconomía, como es el caso del Tecnologías Agroalimentarias y Bioenergías o Agricultura Sostenible. Sus líneas de investigación abarcan desde la mejora y optimización de recursos para la producción, vegetal o animal, hasta aspectos económicos - legislativos. Debido al amplia temática que representa la Bioeconomía, se presentan múltiples áreas en las que los investigadores desarrollan su actividad y son elemento clave para la innovación en la materia, tales como: biodiversidad y medio Ambiente, bioenergía, calidad y seguridad Alimentaria, tecnología de los alimentos, mejora producción, nutrición, sanidad o valorización de subproductos.

La transferencia es uno de los principios fundacionales clave del ceiA3. El campus se define como un elemento de cohesión entre la comunidad científica, la Administración y la industria del sector, promoviendo el desarrollo de múltiples actividades conjuntas en el ámbito agroalimentario. Asimismo cabe 
destacar la participación en proyectos y alineamiento de las investigaciones con las temáticas incluidas bajo el concepto de Bioeconomía llevadas a cabo en los últimos años y la prospección a futuro donde cabe resaltar el especial experiencia en el ámbito de proyectos innovadores tanto regionales como nacionales de GO que tienen como principal fin la innovación vinculada a los sistemas agroalimentarios sostenibles y promover los territorios rurales mediante la colaboración entre agentes del sector y del conocimiento.

Tanto a nivel del propio consorcio ceiA3 como a nivel de investigadores del ceiA3 se ha participado en una veintena de proyectos relacionados con la Bioeconomía, tanto en su reto específico de H2020, como en otros programas Europeos tales como el Programa LIFE, Coal and Steel, 7 Programa Marco o diferentes ERA-NETs.

Entre las actuaciones de apoyo, impulsadas por el CEl, relacionadas con el emprendimiento, se convocan anualmente los premios A3BT para recompensar las mejores ideas y proyectos de empresa

Por último, tanto el consorcio como las propias instituciones integrantes del ceiA3, cuentan con Unidad de Cultura Científica reconocida UCC para dar soporte a la divulgación científica y desarrollar acciones destinadas a la sociedad. 
Figura 1. Esquema del potencial en I+D+i y transferencia del ceiA3 y alineamiento con políticas.

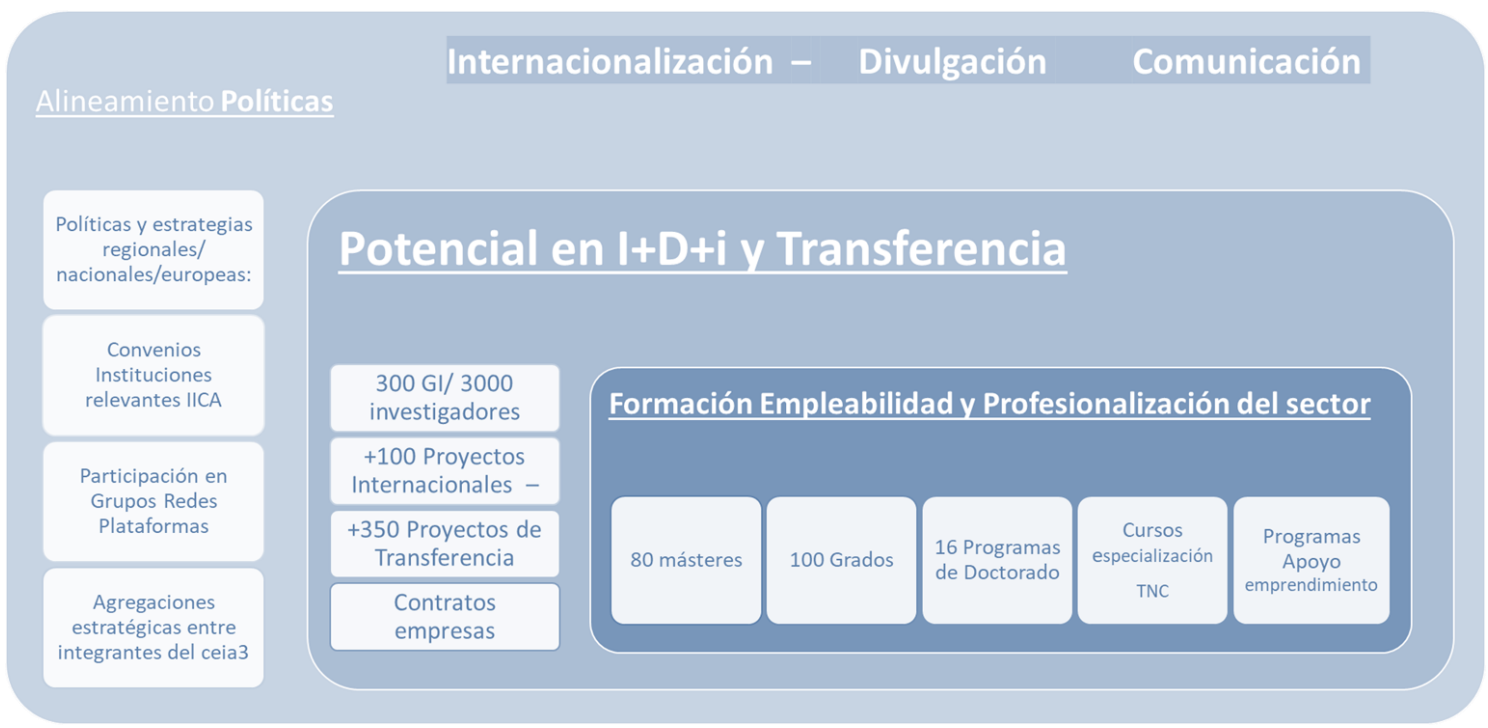

\section{APROXIMACIÓN A LA ESTRATEGIA DE BIOECONOMÍA DEL CAMPUS DE EXCELENCIA}

\subsection{Justificación de la estrategia del ceiA3 en materia de la Bioeconomía}

Tras la primera aproximación al marco conceptual las distintas estrategias, todas ellas coinciden en que el reto es desarrollar sistemas productivos de base biológica sostenibles basados es la circularidad $y$, tanto por su peso medioambiental como por su relevancia económica y social, se presta especial atención al sector agroalimentario como uno de los principales sistemas productivos a tener en cuenta en el territorio europeo, nacional y regional.

En un contexto donde investigación e innovación cobran especial importancia en la implantación de soluciones innovadoras para promover la producción mediante sistemas sostenibles donde se incida en el uso sostenible y recirculación de los recursos biológicos y se minimicen los residuos, el desarrollo de nuevas cadenas de valor basadas en la producción de bioproductos o se aumente el valor añadido de las mismas mediante el uso de los subproductos, las universidades y centros de innovación que cuentan con avalada 
experiencia en el desarrollo de líneas juegan un rol estratégico apoyando a las empresas en la consecución de estos objetivos y favorecer la innovación sostenible de los sistemas productivos.

La innovación precisa el acompañamiento de los sistemas productivos de una formación especializada y capacitación en materia de bioeconomía que se plantea asimismo como una necesidad clave para favorecer los ecosistemas de innovación y la profesionalización del sector. En este ámbito el apoyo a nuevas empresas que apoyen al proceso de innovación se considera asimismo fundamental.

Todo ello debe ir acompañado de la concienciación y coparticipación en el diseño de acciones de todos los actores de la cadena incluyendo a consumidores y la propia sociedad que son un factor fundamental para garantizar a medio y largo plazo el éxito de las medidas implementadas mediante un proceso de diseño "bottom-up".

En la Comunidad andaluza se destaca en su estrategia de especialización inteligente, RIS3 que entre las ventajas regionales de apoyo a la "Investigación e innovación en agroindustria y alimentación saludable" destaca que la región cuenta con el ceiA3 como activo de referencia para la promoción de sinergias entre actores.

La Organización para la Cooperación y el Desarrollo Económicos (OCDE) en 2016 realizó un riguroso informe sobre las marco político del "triángulo del conocimiento" para la integración de la investigación, la educación y la innovación de analizando dieciséis estudios de caso de países sobre ecosistemas de educación superior en el triángulo del conocimiento en Europa, "the knowledge triangle: draft synthesis report" (OCDE, 2016), analizando como caso en relevante en España el del ceiA3 y destacando que favorece la creación de comunidades orientadas a la excelencia en dominios de conocimiento, cumpliendo los objetivos de especialización y, por tanto, la internacionalización de sus actividades. 
En la Estrategia Andaluza de Bioeconomía Circular se reconoce al ceiA3 como entidad clave en el ámbito de la I+D+i+F asociado a la bioeconomía en Andalucía señalando al ceiA3 y sus grupos de investigación como una de las fortalezas del sistema. Así mismo destaca en lo relativo a la innovación "precisamente en términos de innovación, tal y como menciona el Acuerdo de formulación de la Estrategia Andaluza de Bioeconomía Circular, Andalucía cuenta con Universidades, Campus de Excelencia Internacional (ceiA3 ...), Centros y Grupos de Investigación y Formación, tanto públicos como privados, que pueden proporcionar recursos y materiales a la vez que un entorno adecuado para favorecer la investigación y la transferencia de técnicas innovadoras en todos los ámbitos", lo que alentará al cambio necesario (Junta de Andalucía, 2018).

Es por ello por lo que, en ese contexto y alineado con todas estas políticas y estrategias, el órgano de gobierno del ceiA3, el Consejo Rector aprueba el 27 de febrero de 2018 la bioeconomía como línea estratégica transversal del campus y desde entonces se trabaja para favorecer la formación y la transferencia del conocimiento e innovación de sus grupos a través de acciones y proyectos de I+D+i que refuerzan la colaboración de los agentes de la cuádruple hélice en el ámbito de la Bioeconomía, la divulgación científica y la concienciación de la Sociedad y apoyar a un sector agroalimentario innovador, circular y sostenible.

\subsection{Objetivos de la estrategia}

La Bioeconomía, vinculada con sistemas productivos agroalimentarios sostenibles, y en el actual contexto ambiental y político debe ser una línea transversal de priorización para el campus hacia donde se dirigen las investigaciones de sus grupos confiriendo una mayor especialización de los actores, esta materia se desarrolla en la formación y las actuaciones del propio ceiA3, transversalizando en todas sus áreas la Bioeconomía como prioridad. 
Por ello lo anteriormente expuesto y atendiendo a los principios del propio CEl se diseña la estrategia de Bioeconomía del ceiA3 como una línea transversal de especialización hacia destinar especial esfuerzo y siendo sus principales objetivos:

Favorecer el alineamiento de todas las áreas y actuaciones de los integrantes del CEl con las políticas y estrategias regionales, nacionales y europeas en materia de Bioeconomía.

Promover y fortalecer las acciones conjuntas en I+D y la transferencia de conocimiento cuyos fines se alineen con una producción agroalimentaria sostenible.

Reforzar y promover formación y capacitación, la profesionalización del sector y la empleabilidad de egresados de Universidades ceiA3 en Bioeconomía.

Visibilizar y posicionar la investigación e innovación de excelencia llevada a cabo en las universidades y centros asociados del ceiA3, promover la divulgación y la concienciación de la sociedad.

Figura 2. Esquema de los objetivos del ceiA3
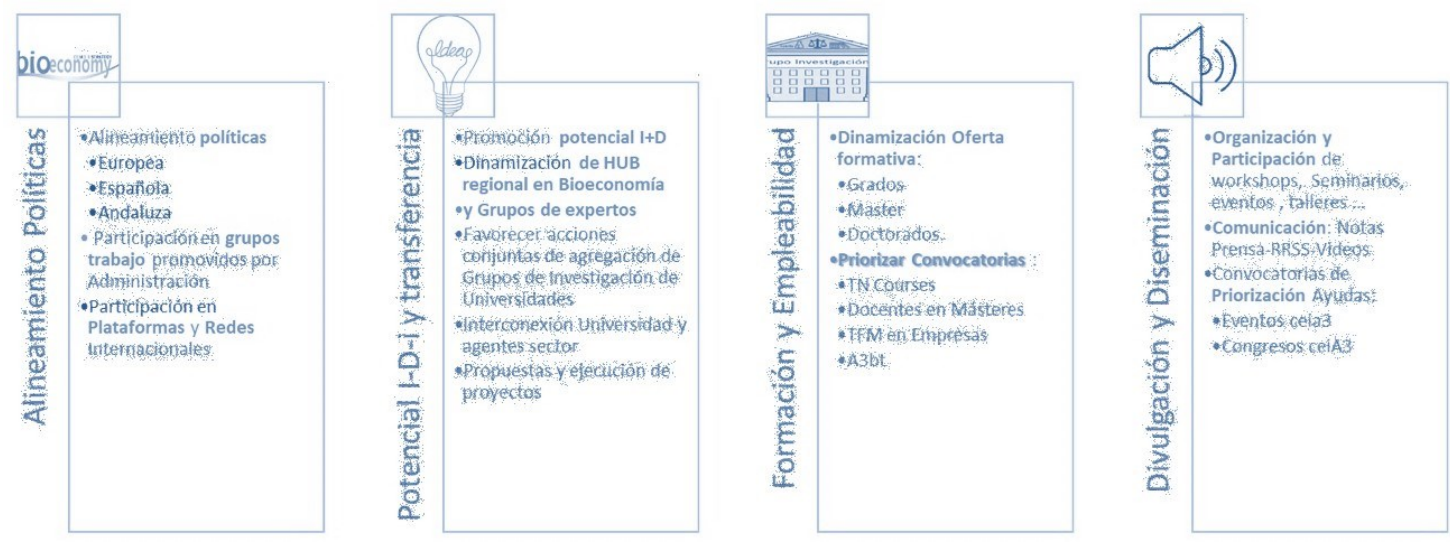


\subsection{Líneas de acción y casos prácticos en el ámbito de la Bioeconomía}

En línea con los principios de agregación de instituciones y especialización se plantea desarrollar una serie de acciones que acometerán de manera transversal las diferentes áreas del campus y que, en último término, ayudarán a la consecución de los objetivos de la estrategia.

Se destacan los ejemplos más relevantes de acciones a ejecutar en cada uno de los bloques propuestos:

En lo relativo a reforzar el alineamiento del ceiA3 con políticas, iniciativas y estrategias regionales, nacionales e internacionales en materia de Bioeconomía se destaca como caso de éxito la participación activa del campus en las correspondiente estrategias nacional y regional a través de la participación en el Observatorio Español de Bioeconomía y en el diseño de la Estrategia Andaluza de Bioeconomía circular tanto por parte de investigadores ceiA3 como del propio campus aunando y representando los intereses de sus instituciones integrantes y aportando su experiencia en la materia.

Asimismo en este bloque se destaca, por su importancia para el sector agroalimentario, el alineamiento del ceiA3 con la iniciativa europea eip-Agri que se materializa a través de la participación en eventos y actividades vinculadas con la Bioeconomía como grupos focales, puesta en marcha de Grupos Operativos y ejecución de proyectos innovadores todo ello ligado a una producción sostenible así como con el compromiso Green Deal y el Horizonte Europa: promoviendo la participación en proyectos consorciados alineados con esta temática

Con el objeto de promover una oferta docente ceiA3 en materia de bioeconomía se plantea como acción a destacar reforzar la implementación de contenidos docentes en la materia, visibilizar la oferta de grados másteres y 
programas de doctorados, promover la formación especializada en Bioeconomía como los Training Network Courses o la priorización en las líneas de ayuda de esta línea estratégica.

La dinamización del potencial de 1+D+i de los Grupos de investigación adscritos al ceiA3 en materia de Bioeconomía es una acción clave que se materializará a través la actualización y dinamización de la capacidad en el ámbito de la investigación e innovación de los grupos en el área de Bioeconomía, la categorización del potencial, la digitalización de la información mediante una plataforma que facilite la búsqueda de capacidades y la constitución de Grupos de Expertos en Bioeconomía.

En línea con el objetivo anterior pero orientado a la promoción de la transferencia y vínculo con el sector se dinamizarán la puesta en marcha de encuentros, talleres y proyectos en esta área, la identificación de sinergias y búsqueda de soluciones innovadoras a las problemáticas del sector acometidas con enfoque multiactor y diseño "bottom-up" y búsqueda de posibles fuentes de financiación públicas y privadas. Todo ello culminará con la ejecución de proyectos innovadores en el marco de diferentes convocatorias como las de grupos operativos de la eip-Agri o proyectos de transferencia e innovación financiados con fondos europeos. Resaltar el proyecto regional PAIDI de transferencia Agroindustria 4.0 y Bioeconomía que ha dado el soporte financiero para el desarrollo de la estrategia.

Por último, dentro del bloque de acciones orientadas a dar visibilidad y divulgación de la información, se hará a través de la Unidad de Cultura científica del ceiA3 y mediante la generación de contenidos, noticias y videos divulgativos. En este bloque destacan las acciones ejecutadas en el marco del Proyecto europeo del programa H2020 BLOOM cuyo objetivo es la concienciación de la sociedad sobre la importancia de la Bioeconomía y donde el ceiA3 participa como responsable del desarrollo de la plataforma web del proyecto y el coordinador responsable de dinamizar el hub español. En el 
marco del hub español en Bioeconomía, de especialización en Agroalimentación, y dinamizado por el ceiA3, tras un mapeo de actores relevantes en la región se implicaron expertos de las universidades, agentes del sector productivo y del territorio y a las propias administraciones. El hub se constituye como grupo multiactor y su principal cometido ha sido el co-diseño, mediante metodología de co-creación de diferentes actividades "outreach" diseñadas para promover la concienciación y destinadas a la sociedad y con especial atención al sector productor y a los grupos de escolares. Las principales actuaciones promovidas han sido desarrollo de materiales para profesorado, participación en eventos como noche de investigadores, rutas de innovación demostrativas a empresas de alta concienciación en materia de bioeconomía y el desarrollo de contenidos y material audiovisual de apoyo.

Finalmente destaca la reflexión sobre la importancia de divulgar los conocimientos científicos innovadores que motivó la puesta en marcha de la Revista científico-técnica C3-Bioeconomy para promover la divulgación, tanto científica como técnica, de innovaciones, experiencias y logros del tejido del conocimiento y tejido productor agroalimentario en materia de bioeconomía.

\section{REFERENCIAS}

Organización de las Naciones Unidas para la Alimentación y la Agricultura, FAO (2018). Cambio climático. Recuperado de: http://www.fao.org/climate-change/our-work/issues/bioeconomy/es/.

Comunicación de la Comisión al Parlamento Europeo, al Consejo, al Comité Económico y Social Europeo y al Comité de las Regiones (2018). Recuperado de: https://ec.europa.eu/transparency/regdoc/rep/1/2018/ES/COM2018-673-F1-ES-MAIN-PART-1.PDF . 
Comunicado Cumbre Global de Bioeconomía 2018. Global Bioeconomy Summit 2018. Recuperado de:

https://gbs2018.com/fileadmin/gbs2018/Downloads/Communique\%C C\%81GBS2018 final Spanish.pdf.

The knowledge triangle: draft synthesis report. Organización de Cooperación y Desarrollo Económico, OCDE (2016). Recuperado de: https://one.oecd.org/document/DSTI/STP/TIP(2016)10/en/pdf.

Junta de Andalucía (2018). Estrategia Andaluza de Bioeconomía Circular. Recuperado de: https://www.juntadeandalucia.es/export/drupaljda/Estrategia Andaluz a_Bioeconomia_Circular_EABC_18.09.2018.pdf.

Boosting European Citizen's knowledge and awareness of Bio-Economy Research and Innovation. Recuperado de: https://bloombioeconomy.eu/. 